\title{
Geometrical Interpretation of the Bilinear Equations for the KP Hierarchy
}

G. F. HEL MINCK and G, F, POST

University of Twente. Faculty of Applied Mathematics, P.O. Box 217,7500 AE Enschede, The Netherlands

(Received: 25 May 1988)

Abstract. The Grassmann manifold approach to the KP hierarchy, in the spirit of Segal and Wilson, is used to define the wavefunction $\psi_{w}$ and its adjoint $\psi_{w}$. From the fact that $\psi_{w}$ and $\psi_{w \perp}$ are the orthogonal, we derive the bilinear equations. The modified equations are treated at the same time.

\section{Introduction}

In this Letter we give a geometrical interpretation of the bilinear form of the equations of the KP hierarchy and its modified versions. Apart from [1], this formula is derived using generalized Plucker coordinates $[2,3,4]$. (Only [3] contains the modified versions.)

Now one can associate to each subspace $W$ in the Grassmann manifold of $L^{2}\left(S^{\prime}, C\right)$ a wavefunction $\psi_{W}$ which is a solution of the linear system connected to the KP equations. In a natural way, we will link a wavefunction $\psi_{W \perp}$ to the orthocomplement $W \perp$ of $W$. Expressing $\psi_{W}$, in a $\tau$-function, we prove that the bilinear equations boil down to the orthogonality relations for $\psi_{w_{1}}$ and $\psi_{W_{\frac{1}{2}}}$ for $W_{1} \subset W_{2}$.

Contrary to $[1-4]$, our framework is analytic.

\section{The Grassmann Manifold and Wavefunctions}

Let $H$ be $L^{2}\left(S^{1}, \mathbb{C}\right)$. The Fourier series of $f \in H$ we denote by $\Sigma_{i \in \mathbb{Z}} a_{i} \lambda^{i}, a_{i} \in \mathbb{C}$. For each $l$ in $\mathbb{Z}$, we write

$$
H_{l}=\left\{\sum_{i \geqslant i} a_{i} \lambda^{i} \in H\right\} .
$$

The spaces $H_{0}$ and $H_{0}^{\perp}$ are also denoted by $H_{+}$, resp. $H_{-}$, as in $[5,6]$. To the decomposition $H=H_{+} \oplus H_{-}$is associated the Grassmann manifold $\operatorname{Gr}(H)$ of $H$. It is a homogeneous space of the group $\mathrm{Gl}_{\text {res }}(H)$, which consists of the automorphisms $g$ of $H$ that decompose w.r.t. $H=H_{+} \oplus H_{-}$as

$$
g=\left(\begin{array}{ll}
a & b \\
c & d
\end{array}\right), \quad a \text { and } d \text { Fredholm, } b \text { and } c \text { Hilbert-Schmidt }
$$


The connected components $\mathrm{Gr} r^{(i)}(H), l \in \mathbb{Z}$, are determined by

$$
\begin{aligned}
\mathrm{Gr}^{(l)}(H) & =\left\{\left(\begin{array}{ll}
a & b \\
c & d
\end{array}\right)\left(H_{+}\right) \mid \text {index }(a)=l\right\}=\mathrm{Gl}_{\mathrm{res}}^{l} \cdot H_{+} \\
& =\left\{\left(\begin{array}{ll}
a & b \\
c & d
\end{array}\right)\left(H_{l}\right), \text { index }(a)=0\right\} .
\end{aligned}
$$

As in [5], one shows that $\mathrm{Gl}_{\text {res }}^{0}(H)$ acts transitively on each of the $\mathrm{Gr}^{(\ell)}(H)$.

Let $\Lambda: H \rightarrow H$ be the 'multiplication by $\lambda$ '. The group of flows connected to the KP hierarchy is

$$
\Gamma_{+}=\left\{\exp \left(\sum_{i \geqslant 1} t_{i} \Lambda^{i}\right) \in \mathrm{Gl}_{\mathrm{res}}^{0}(H), \sum_{i \geqslant 1}\left|t_{i}\right|(1+\varepsilon)^{i}<\infty \text { for some } \varepsilon>0\right\} .
$$

For each $W_{l}$ in $\operatorname{Gr}^{(l)}(H)$, let $\Gamma_{+}^{W_{l}}$ be given by

$$
\Gamma_{+}^{W_{l}}=\left\{\gamma \in \Gamma_{+} \mid \text {orthogonal projection } \eta^{-1} W_{l} \rightarrow H_{l} \text { is a bijection }\right\} .
$$

As in [6], one shows that $\Gamma_{+}^{W_{l}}$ is nonempty. To $W_{l}$ one associates a wavefunction $\psi_{W_{l}}(\gamma, \zeta)$, where $\gamma \in \Gamma_{+}^{W_{I}}$ and $1<|\zeta|<1+\varepsilon$, of the form

$$
\begin{aligned}
\psi_{W_{l}}(\gamma, \zeta) & =\zeta^{l}\left(1+\sum_{k>0} a_{k}(\gamma) \zeta^{-k}\right) \exp \left(\sum_{i \geqslant 1} t_{i} \zeta^{i}\right) \\
& =\hat{\psi}_{W i}(\gamma, \zeta) \exp \left(\sum_{i \geqslant 1} t_{i} \zeta^{i}\right) \\
& =\zeta^{l}\left\{\sum_{j \in \mathbb{Z}} \alpha_{j}(\gamma) \zeta^{j}\right\},
\end{aligned}
$$

which has the property that its $L^{2}$-boundary value $\psi_{W_{l}}(\gamma, \lambda), \lambda \in S^{1}$, belongs to $W_{l}$ for all $\gamma$ in $\Gamma_{+}^{W_{t}}$. The wavefunction is uniquely determined by this requirement.

Next, we introduce the adjoint wavefunction of $\psi_{w}$. A direct verification shows that if $W_{l} \in \mathrm{Gr}^{(i)}(H)$, then the orthogonal projection of $W_{l}^{\perp}$ to $H_{-}$is a Fredholm operator of index $-l$.

Moreover, we have for each $\gamma$ in $\Gamma_{+}$and $W_{l} \in \mathrm{Gr}^{(l)}(H)$ that $\left(\gamma^{-1} W_{l}\right)^{\perp}=\left(\gamma^{*}\right) W_{l}^{\perp}$, where $\gamma^{*}$ is the adjoint of $\gamma$. Now we exchange the role of $H_{+}$and $H_{-}$and the Grassmann manifold connected to this decomposition of $H$ contains all $W_{l}^{\perp}$. On the new Grassmann manifold we consider the adjoint flow-group, i.e.

$$
\Gamma_{-}=\left\{\exp \left(\sum_{i \geqslant 1} s_{i} \Lambda^{-i}\right) \in \mathrm{Gl}_{\mathrm{res}}^{0}(H), \sum_{i \geqslant 1}\left|s_{i}\right|(1+\varepsilon)^{i}<\infty \text { for some } \varepsilon>0\right\}
$$

and introduce analogously

$$
\begin{aligned}
\Gamma_{-}^{W_{l}^{\perp}} & =\left\{\gamma \in \Gamma_{-}, \text {the orthogonal projection } \gamma_{1}^{-1} W_{l}^{\perp} \rightarrow H_{l}^{\perp} \text { is a bijection }\right\} \\
& =\left\{\left(\gamma^{*}\right)^{-1}, \gamma \in \Gamma_{+}^{W}\right\} .
\end{aligned}
$$


The adjoint wavefunction $\psi_{w_{i} \perp}(\gamma, \eta)$, with

$$
\gamma \in \Gamma_{-}^{W_{t}^{\perp}} \text { and } \frac{1}{1+\varepsilon}<|\eta|<1 \text {, }
$$

has the form

$$
\begin{aligned}
\psi_{w, \perp}(\gamma, \eta) & =\eta^{i-1}\left(1+\sum_{k>0} b_{k}(\gamma) \eta^{k}\right) \exp \left(\sum_{l \geqslant 1} s_{i} \eta^{-i}\right) \\
& =\hat{\psi}_{W_{l}^{\perp}}(\gamma, \eta) \exp \left(\sum_{i \geqslant 1} s_{i} \eta^{-i}\right) \\
& =\eta^{\prime-1} \sum_{j \in \mathbb{Z}} \beta_{j}(\gamma) \eta^{j}
\end{aligned}
$$

and is uniquely determined by the property that the $L^{2}$-boundary value $\psi_{W_{i}}\llcorner(\gamma, \lambda)$, $\lambda \in S^{\mathbf{1}}$, belongs to $W_{l}^{\perp}$ for all $\mathrm{y}$ in $\Gamma_{-}^{W_{l}^{\perp}}$.

\section{The Bilinear Equations}

For $k$ and $l \in \mathbb{Z}, k \geqslant l$, the Japanese school discussed in [3] the bilinear equations of the $(k, l)$ modified KP hierarchy. We will show that these relations are nothing but the following observation: Assume $W_{l} \in \mathrm{Gr}^{(l)}(H)$ and $W_{k} \in \mathrm{Gr}^{(k)}(H)$ are such that $W_{l} \supseteq W_{k}$. This happens, e.g., for $W_{l}=g \cdot H_{l}$ and $W_{k}=\mathrm{g} \cdot H_{k}$ with $g \in \mathrm{Gl}_{\mathrm{res}}^{0}(H)$. Then $W_{l}{ }^{1} \subset W_{k}^{\perp}$ and due to the defining properties of $\psi_{W_{i}{ }^{\perp}}$ and $\psi_{W_{k}}$, namely $\psi_{W_{k}}(\gamma, \lambda) \in W_{k}$ and $\psi_{W F^{\perp}}(\gamma, \lambda) \in W_{l}^{\perp}$, we have

$$
\begin{gathered}
\int_{|\lambda|=1} \psi_{W_{k}}(\gamma, \lambda) \overline{\psi_{W_{j}^{\prime}}(\gamma, \lambda)} \mathrm{d} \lambda=0 \\
=\sum_{j \in \mathbb{Z}} \alpha_{j}(\gamma) \bar{\beta}_{j+1+k-l}(\gamma)=0 .
\end{gathered}
$$

We rewrite (1) such that the integrand becomes analytic in $\lambda$. For

$$
\overline{\psi_{W_{f}^{\perp}}(\gamma, \lambda)}=\lambda^{1-l}\left(1+\sum \bar{b}_{k}(\gamma) \lambda^{-k}\right) \exp \left(\sum_{i \geqslant 1} \bar{s}_{i} \lambda^{i}\right)
$$

Thus (1) becomes

$$
\oint \lambda^{k-i}\left(1+\sum_{i \geqslant 1} a_{i}(\gamma) \lambda^{-i}\right)\left(1+\sum_{j \geqslant 1} \bar{b}_{j}(\gamma) \lambda^{-j}\right) \exp \left(\sum_{i \geqslant 1}\left(t_{i}+\bar{s}_{t}\right) \lambda^{i}\right) \mathrm{d} \lambda
$$

with $\mathrm{d} \lambda$ such that for analytic $f$ in $1<|\lambda|<1+\varepsilon$

$$
\oint f(\lambda) \mathrm{d} \lambda=\operatorname{Res}(f)_{\lambda=0} \text {. }
$$


Next we give the expression of $\hat{\psi}_{w_{l}}$ and $\hat{\psi}_{w_{l}{ }^{1}}$ in terms of the $\tau$-function associated to $W_{l}$. Substituting this into (2) gives you the formula (2.4) occurring in [3]. Let $w_{l}$ be a continuous embedding of $H_{l}$ into $H$ with image $W_{l} \in \mathrm{Gr}^{(l)}(H)$ and denote its decomposition w.r.t. $H=H_{l} \oplus H_{i}^{\perp}$ by $\left(\begin{array}{c}\left(w_{i}\right)_{+} \\ \left(w_{l}\right)_{-}\end{array}\right)$. As shown in [5], we may assume that $\left(w_{l}\right)_{+}$has the form 'identity + trace-class'. To such an embedding we associate a function $\tau_{w r}$ on $\Gamma_{+}$by

$$
\tau_{w_{l}}(\gamma)=\tau_{w_{l}}\left(\exp \left(\sum_{i>0} t_{i} \Lambda^{i}\right)\right):=\operatorname{det}\left(\left(w_{i}\right)_{+}+a^{-1} b\left(w_{i}\right)_{-}\right)
$$

where $\left(\begin{array}{cc}a & b \\ 0 & d\end{array}\right)$ is the decomposition of $\gamma^{-1}$ w.r.t. $H_{l} \oplus H_{l}^{\perp}$. Using the multiplicativity of det, one shows that if the orthogonal projection of $\gamma_{1}^{-1} W_{l}$ to $H_{i}$ is bijective then

$$
\frac{\tau_{w_{t}}\left(\gamma_{1} \gamma_{2}\right)}{\tau_{w / t}\left(\gamma_{1}\right)}=\tau_{w,\left(l_{1} \gamma_{1}\right)}\left(\gamma_{2}\right) \quad \text { with } \quad w\left(l_{1} \gamma_{1}\right)=\left(\begin{array}{c}
\text { Id } \\
\left(y^{-1} \circ w_{l}\right)_{-} \circ\left(\gamma_{1}^{-1} \circ w_{l}\right)_{+}^{-1}
\end{array}\right) .
$$

For $|\zeta|>1$, let $q_{\zeta}$ be

$$
1-\frac{\Lambda}{\zeta}=\exp \left(-\sum_{i \geqslant 1} \frac{1}{i \zeta^{i}} \Lambda^{i}\right) \in \Gamma_{+} .
$$

Let $\left(\begin{array}{ll}a & b \\ 0 & d\end{array}\right)$ be its decomposition w.r.t. $H_{l} \oplus H_{i}{ }^{\perp}$. Then a direct computation shows that $b$ is given by: for all $t<l$

$$
\lambda^{t}+\zeta^{t} \cdot \sum_{k=l}^{\infty}\left(\frac{\lambda}{\zeta}\right)^{k}
$$

Hence, $a^{-1} b$ maps $\Sigma_{t<l a_{t} \lambda^{2}}$ to $\left\{\Sigma_{t<i} a_{t} \zeta^{t}\right\} \cdot \lambda^{\prime} / \zeta^{t}$. Now we apply formula (3) with $\gamma_{1} \in \Gamma_{+}$and $\gamma_{2}=q_{\zeta}$. Denote for simplicity $\left(\gamma_{1}^{-1} \circ w_{l}\right)_{-} \circ\left(\gamma_{1}^{-1} \circ w_{l}\right)_{+}^{-1}$ by $w_{-}$. By definition, we know that

$$
\hat{\psi}_{w_{l}}\left(\gamma_{1}, \lambda\right)=\lambda^{l}+w_{-}\left(\lambda^{l}\right)=\lambda^{l}+\sum_{k<l} w_{k l}\left(\gamma_{1}\right) \lambda^{k} .
$$

Now $\tau_{w}\left(q_{\zeta}\right)=1+\operatorname{Trace}\left(a^{-1} b w_{-}\right)=1+$ coefficient of $\lambda^{l}$ in $\left(a^{-1 b w_{-}}\right) \lambda^{l}=$ $1+\xi^{-l} \Sigma_{k<l} w_{k i}\left(\gamma_{1}\right) \xi^{t^{k}}$. Thus, we get

$$
\hat{\psi}_{w_{l}}\left(\gamma_{1}, \zeta\right)=\zeta^{i} \cdot \frac{\tau_{w_{i}}\left(\gamma_{1} q_{\zeta}\right)}{\tau_{w_{l}}\left(\gamma_{1}\right)}=\zeta^{t} \cdot \frac{\tau_{w_{l}}\left(\exp \left(\Sigma\left(t_{i}-\frac{1}{i} \zeta^{-i}\right) \Lambda^{i}\right)\right)}{\tau_{w,}\left(\exp \left(\Sigma t_{i} \Lambda^{i}\right)\right)} .
$$

The formula (2.4) in [3] already indicates the direction we have to look for in the case of $\hat{\psi}_{W_{l}^{\perp}}$ : instead of $q_{\zeta}$ consider $\left(q_{\zeta}\right)^{-1}$. Therefore, we start with the computation of $\tau_{w}\left(q_{\xi}^{-1}\right)$ with $w=\left(\begin{array}{c}\text { Id } \\ w_{-}\end{array}\right)$. Let $\left(\begin{array}{ll}{ }_{0}^{a} & b \\ d\end{array}\right)$ be the decomposition of $q_{\zeta}$ w.r.t. $H=H_{l} \oplus H_{l}^{\perp}$. Then $b$ is given by

$$
\sum_{j<\lambda} c_{j} \lambda^{j}+-c_{l-1} \cdot \frac{1}{\zeta} \cdot \lambda^{l}
$$


and $a^{-1} b$ by

$$
\sum c_{j} \lambda^{j} \mapsto \frac{-c_{l-1}}{\zeta} \cdot \sum_{k=0}^{\infty} \frac{\lambda^{k+l}}{\zeta^{k}}=\frac{-c_{l-1} \cdot \zeta^{l}}{\zeta} \sum_{m \geqslant l} \frac{\lambda^{m}}{\zeta^{m}} .
$$

In particular, its image is one-dimensional and, therefore,

$$
\operatorname{det}\left(\operatorname{Id}+a^{-1} b w_{-}\right)=1+\operatorname{Trace}\left(a^{-1} b w_{-}\right) .
$$

Now let $\left(w_{s m}\right), s<1$ and $m \geqslant l$, be the matrix coefficients of $w_{-}$w.r.t. the basis $\left\{\partial^{i}\right\}$, i.e.

$$
w_{-}\left(\lambda^{m}\right)=\sum_{s<l} w_{s m} \lambda^{s}, \quad m \geqslant l
$$

Then

$$
a^{-1} b w_{-}\left(\lambda^{m}\right)=-w_{t-1 m} \zeta^{i-1} \sum_{k \geqslant 1} \frac{\lambda^{k}}{\zeta^{k}}
$$

Hence,

and

$$
\operatorname{Trace}\left(a^{-1} b w_{-}\right)=\left\{\sum_{m \geqslant l}-w_{l-1 m} \zeta^{-m}\right\} \zeta^{l-1}
$$

$$
\tau_{w}\left(q_{\zeta}^{-1}\right)=1+\left\{\sum_{m \geqslant l}-w_{l-1 m} \zeta^{-m}\right\} \zeta^{l-1} .
$$

If $\gamma \in \Gamma_{+}^{W_{I}}$, then $\gamma=\left(\gamma^{*}\right)^{-1} \in \Gamma_{-}^{W_{l}}$. Let $w_{-}$be $\left(\gamma^{-1} \circ w_{l}\right)_{-}{ }^{\circ}\left(\gamma^{-1} \circ w_{l}\right)_{+}^{-1}$ then one verifies directly that $\left(\left(^{-\left(w_{+}\right)^{*}}\right)\right.$ is an embedding of $H_{l}^{\perp}$ into $H$ with image $\gamma^{*} W_{l}^{\perp}$. Hence, by definition

$$
\psi_{W_{i}^{\perp}}(\gamma, \lambda)=\lambda^{I-1}+\left\{\left(-w_{-}\right)^{*}\right\}\left(\lambda^{l-1}\right) .
$$

Let $\left(w_{s m}\right)$ be as above then the $(m, s)$ th matrix coefficient of $\left(-w_{-}\right)^{*}$ is $-\bar{w}_{s m}$. In particular, we have

Therefore,

$$
\left(-w_{-}\right) *\left(\lambda^{I-1}\right)=\sum_{m \geqslant i}-\bar{w}_{t-1 m} \lambda^{m}
$$

$$
\psi_{W_{i} \mathrm{~L}}(\gamma, \eta)=\eta^{l-1}+\sum_{m \geqslant l}-\bar{w}_{l-1 m}(\gamma) \eta^{m}
$$

Hence, in the integrand of (2), we get

$$
\begin{gathered}
\zeta^{\mathbf{l}-I}+\sum_{m \geqslant l}-w_{l-1 m}(\gamma) \zeta^{-m}=\zeta^{1-1} \tau_{w}\left(q_{\zeta}^{-1}\right) \\
=\zeta^{1-1} \frac{\tau_{w}\left(\gamma q \zeta^{-1}\right)}{\tau_{w^{\prime}}(\gamma)}=\zeta^{1-1} \frac{\tau_{w}\left(\left(t_{i}+\frac{1}{i} \zeta^{-1}\right)\right)}{\tau_{w}(\gamma)} .
\end{gathered}
$$


Collecting all the expressions computed above, we have exactly formula (2.4) $)_{k, t}$ in [3] Indeed, if $\gamma^{\prime}=\exp \left(\Sigma t_{i}^{\prime} \Lambda^{i}\right) \in \Gamma_{+}^{W^{\prime}}$, then $\left(\gamma^{\prime}\right)^{-1}=\exp \left(-\Sigma \bar{t}_{i}^{\prime} \Lambda^{-i}\right)\left(=\exp \left(\Sigma s_{i} \Lambda^{-i}\right)\right.$ above), and (2) becomes, after multiplication by $\tau_{w_{i}}(\gamma) \cdot \tau_{w /}\left(\gamma^{\prime}\right)$,

$$
\begin{aligned}
& \oint \lambda^{k-I} \tau_{w_{k}}\left(\left(t_{i}-\frac{1}{i} \lambda^{-i}\right)\right) \tau_{w_{i}}\left(\left(t_{i}^{\prime}+\frac{1}{i} \lambda^{-i}\right)\right) \exp \left(\sum_{j \geqslant 1}\left(t_{j}-t_{j}^{\prime}\right) \lambda^{j}\right) \mathrm{d} \lambda=0, \\
& (k, l \in \mathbb{Z}, k \geqslant l) .
\end{aligned}
$$

\section{References}

1. Date, E., Jimbo, M., Kashiwara, M., and Miwa, T., Transformation groups for soliton equations, Proc. RIMS Sympos. Non-Linear Integrable Systems - Classical Theory and Quantum Theory, World Science Publ., Singapore, 1983.

2. Sato, M. and Sato, Y., in Lecture Notes in Num. Appl. Anal. 5, 1982.

3. Jimbo, M. and Miwa, T., Solitons and Infinite Dimensional Lie Algebras, RIMS-439, Kyoto University, 1983.

4. Kac, V. G., Infinite Dimensiona! Lie Algebras, Progress in Math. Vol. 44, Birkhäuser, Basle, 1983.

5. Pressley, A. and Segal, G., Loop Groups, Oxford, 1986.

6. Segal, G. and Wilson, G., Loops groups and equations of KdV type, Pubi. Math. IHES 61, 1985. 